\title{
Enhanced resistance of single-layer graphene to ion bombardment
}

\author{
J. J. Lopez, ${ }^{1}$ F. Greer, ${ }^{2}$ and J. R. Greer,a) \\ ${ }^{1}$ East Los Angeles College, California 91754, USA \\ ${ }^{2}$ Jet Propulsion Laboratory, California 91109, USA \\ ${ }^{3}$ Division of Engineering and Applied Science, California Institute of Technology, California 91125, USA
}

(Received 17 January 2010; accepted 15 April 2010; published online 27 May 2010)

\begin{abstract}
We report that single-layer graphene on a $\mathrm{SiO}_{2} / \mathrm{Si}$ substrate withstands ion bombardment up to $\sim 7$ times longer than expected when exposed to focused $\mathrm{Ga}^{+}$ion beam. The exposure is performed in a dual beam scanning electron microscope/focused ion beam system at $30 \mathrm{kV}$ accelerating voltage and $41 \mathrm{pA}$ current. $\mathrm{Ga}^{+}$ion flux is determined by sputtering a known volume of hydrogenated amorphous carbon film deposited via plasma-enhanced chemical vapor deposition. () 2010 American Institute of Physics. [doi:10.1063/1.3428466]
\end{abstract}

\section{INTRODUCTION}

Graphene, a one atom thick sheet of $s p^{2}$ hybridized carbon atoms arranged in a honeycomb lattice, is highly researched because of its unique and advantageous electronic properties. ${ }^{1-4}$ The combination of its ballistic electron transport, ${ }^{5}$ high elastic limit, ${ }^{6}$ and the recent achievement of producing macroscale conducting sheets ${ }^{7}$ makes its use in future nanoelectronic devices promising. Due to the challenges associated with manipulating and producing these nanoscale specimens, multiple forms of characterization are required, including but not limited to optical microscopy, atomic force microscopy, Raman spectroscopy, scanning electron microscopy (SEM), and transmission electron microscopy. These techniques utilize different mechanisms to determine material properties and, consequently, have different interactions with the graphene lattice. Recent investigations demonstrate that defects can be introduced into the graphene lattice via electron beam irradiation as manifested by the appearance of a disorder-related $\mathrm{D}$ peak and a change in height of both the D peak and zone-center G peak within graphene's Raman spectra. ${ }^{8}$ It is, therefore, likely that particle bombardment-electronic or ionic — causes varying degrees of damage in graphene. Therefore, in order to design and maintain graphene's desired characteristics, it is critical to understand its response to electron and ion bombardment. Here we report that a single-layer graphene sheet is approximately seven times more resilient against ion bombardment than expected based on sputtering mechanisms of other carbon-based structures.

\section{EXPERIMENT}

Graphene samples were obtained by mechanical exfoliation of highly oriented pyrolitic graphite (SPI Supplies) and subsequently transferred onto $\mathrm{Si}$ substrates with a $300 \mathrm{~nm}$ thick $\mathrm{SiO}_{2}$ layer by using Nitto Denko tape. ${ }^{9,10}$ Single-layer flakes were first identified by optical microscopy (Nikon Eclipse LV100D) using white light to distinguish the graphene sheets via the added optical path that causes an

\footnotetext{
${ }^{a)}$ Author to whom correspondence should be addressed. Electronic mail: jrgreer@caltech.edu.
}

increase in contrast against the $\mathrm{SiO}_{2} / \mathrm{Si}$ substrate. ${ }^{9,11}$ Figure 1 shows optical and SEM images of such representative single-layer sheets. Once the single-layer flakes were identified, Raman spectroscopy with a Reinshaw spectrometer, operating at room temperature with a holographic notch filter (HNF) $514 \mathrm{~nm}$ laser working at $\sim 1.25 \mathrm{~mW}$, was used to confirm their thickness. The Raman spectrum for the sample shown in Fig. 1 with a G peak near $1580 \mathrm{~cm}^{-1}$ and $\mathrm{G}^{\prime}$ band [also known as 2D] near $2700 \mathrm{~cm}^{-1}$ is provided in Fig. 2. The sample is defect-free as indicated by the lack of a disorder-related D peak near $1350 \mathrm{~cm}^{-1}$. The symmetric shape of the 2D peak confirms that the sample is indeed a single sheet, as demonstrated by Ferrari et al. and Gupta et al. ${ }^{12,13}$ Fig. 2(b).

Ion and electron beam imaging was performed in a FEI Nova 200 DualBeam System with a $\mathrm{Ga}^{+}$liquid metal ion source. The e-beam was operated at an acceleration voltage of $10 \mathrm{kV}$ and beam current of $0.54 \mathrm{nA}$. During the initial alignment, the graphene sample was exposed to the e-beam for $\sim 20$ minutes. The sample was then tilted to the eucentric point at a $52^{\circ}$ incline with respect to the e-beam at a working distance of $5.1 \mathrm{~mm}$ to allow simultaneous imaging of the region with both columns. Magnifications and beam positions were coupled to ensure that the graphene sample could be reliably imaged without unnecessary exposure to either beam. After taking the initial SEM image of the graphene sheet, a single ion beam frame was taken at an accelerating voltage of $30 \mathrm{kV}$ and beam current of $41 \mathrm{pA}$ (nominally 10 $\mathrm{pA})$ to intentionally sputter away the single-layer region.

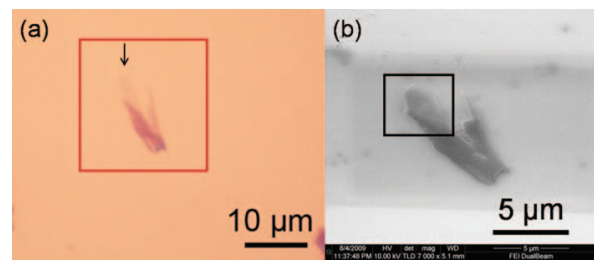

FIG. 1. (Color) (a) Optical image of single-layer graphene in the top left corner of the sample indicated by an arrow. The dimensions of the singlelayer region are $\sim 3 \times 2 \mu \mathrm{m}^{2}$. (b) SEM image of the same sample with single-layer region depicted by the box, taken at $10 \mathrm{kV}$ and $0.54 \mathrm{nA}$. 

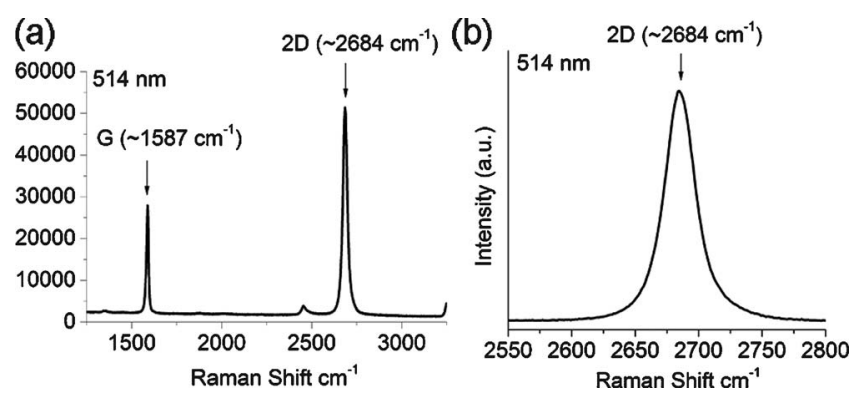

FIG. 2. (a) Raman spectra at $514 \mathrm{~nm}$ for graphene region shown in Fig. 1 (b) $2 \mathrm{D}$ peak at $\sim 2684 \mathrm{~cm}^{-1}$

SEM and focused ion beam (FIB) scans were iteratively repeated until the graphene sheet could no longer be identified in subsequent SEM images, Fig. 3.

We calculate the scan time per frame of $2.7 \mathrm{~s}$ by multiplying the total number of pixels in a single ion beam image $(1024 \times 884)$ by the beam dwell time per pixel $(3 \mu \mathrm{s})$. The graphene sheet was then iteratively sputtered away with each scan. The final step, Figs. 3(d) and 3(i), shows that the region originally covered by single-layer graphene became the same hue as the underlying $\mathrm{SiO}_{2}$. The total time to sputter away this sheet was $57 \mathrm{~s}$, a surprisingly high value for a singleatom-thick material.

To determine whether this etch time was reasonable, we performed a similar experiment on a hydrogenated amorphous carbon (a-C:H) film with an average thickness of 125 nm, Fig. 4(d). Sputtering of this film was also used to calculate the incident $\mathrm{Ga}^{+}$ion flux and subsequently determine the sputtering time of these carbon-based structures. The a-C:H was deposited on a Si substrate via plasma-enhanced chemical vapor deposition with a methane/hydrogen/argon containing plasma. The amorphous and hydrogenated nature of the film was confirmed with Raman spectroscopy under the same conditions as used for graphene. Using a two-peak Gaussian fit for the deconvolution of the D and G peaks, we determined their respective positions as 1371 and $1544 \mathrm{~cm}^{-1}$, as well as the areas under the fitted curves, ${ }^{14}$ Fig. 4(b). By using the ratio of these areas, $I(D) / I(G)$ $=0.79$, and specific position of the $\mathrm{G}$ peak we determined the relative fractions of $s p^{3}$ and $s p^{2}$ hybridized carbon-carbon bonds in the film to be $\sim 38 \% s p^{3}$ and $62 \% s p^{2}$. ${ }^{15}$

\section{RESULTS AND DISCUSSION}

We estimate the sputter yield of this a-C:H film to be $2.63 \mathrm{C}$ atoms/incident $\mathrm{Ga}^{+}$by utilizing the determined

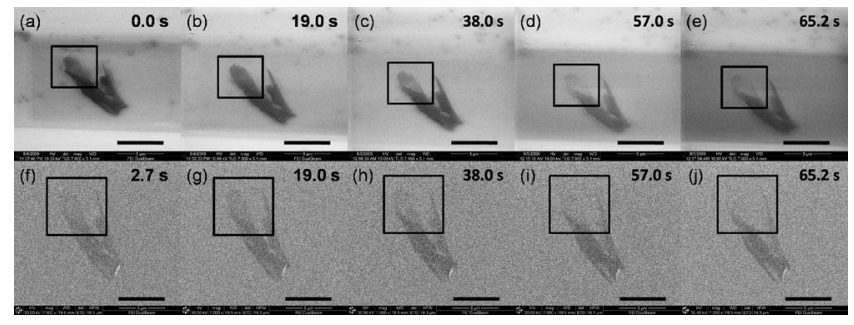

FIG. 3. [(a)-(e)] SEM images of the graphene flake shown in Fig. $1\left(52^{\circ}\right.$ tilt). [(f)-(j)] FIB images of the same flake as a function of exposure (orthogonal). Total area covered by the FIB image is $290.1 \mu \mathrm{m}^{2}$. Scale bar is $5 \mu \mathrm{m}$. Contrast in images was digitally enhanced to accurately identify the time of complete sample sputtering.

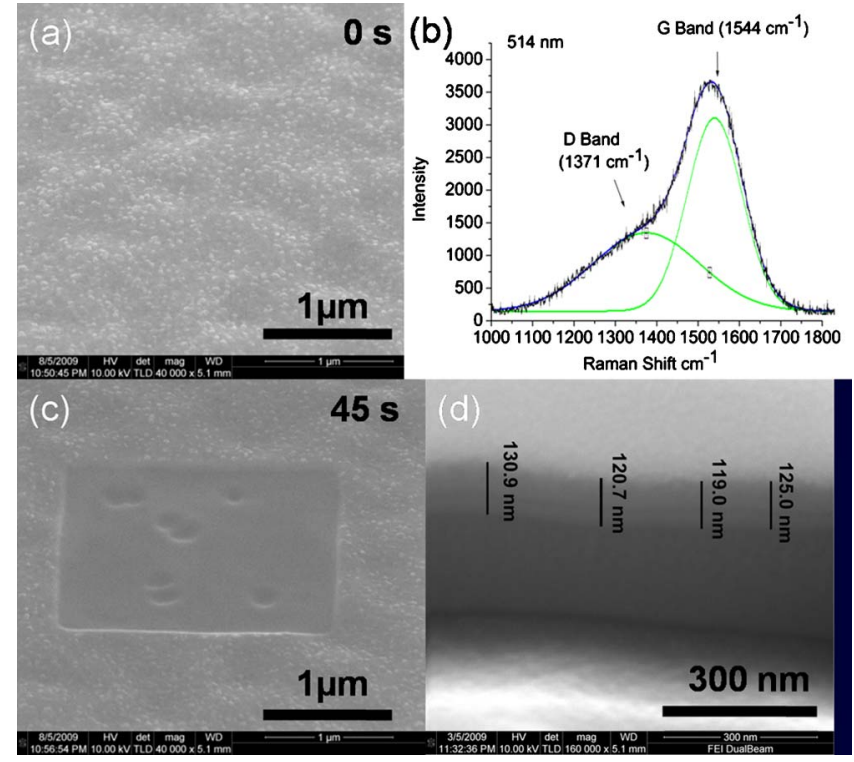

FIG. 4. (Color online) (a) Initial SEM image of the a-C:H film prior to FIB exposure. (b) Raman spectrum of the a-C:H film with D and G peak deconvolution. (c) The darker region is the selected $4.33 \mu \mathrm{m}^{2}$ area of the a-C:H film after $45 \mathrm{~s}$ exposure to ion beam at $30 \mathrm{kV} / 41 \mathrm{pA}$. Wells in the middle of the exposed area shows the $\mathrm{SiO}_{2}$ underneath had been reached. (d) FIBmachined cross-section of the a-C:H film taken in the SEM. Top ( $\sim 200 \mathrm{~nm}$ of $\mathrm{Pt}$ )/middle (125 nm of a-C:H)/bottom (Si substrate). SEM images 4(a), 4(c) $-4(\mathrm{~d})$ are captured while the a-C:H film is at a $52^{\circ}$ incline with respect to the e-beam. All measurements are corrected for the $52^{\circ}$ incline.

weighted average of the $s p^{3}$ and $s p^{2}$ bonds in the film and the reported sputter yields for different carbon allotropes under $\mathrm{Ga}^{+}$ion bombardment: 2.3, 2.55, and 2.7. ${ }^{16}$ The sputter yields at $30 \mathrm{kV}$ are assumed to be the same as those experimentally determined at $50 \mathrm{kV}$ due to flattening out of the yield curve as a function of accelerating voltage at energies of $30 \mathrm{keV}$ and higher. ${ }^{17}$ Figure 4(a) shows the a-C:H film before its exposure to the ion beam, and Fig. 4(c) shows the selected $2.15 \times 2.06 \mu \mathrm{m}^{2}$ area $\left(4.33 \mu \mathrm{m}^{2}\right)$ in the film that is completely sputtered away after $45 \mathrm{~s}$ of exposure to the same ion beam conditions as in the graphene experiments.

Generally, experimentally-determined values for a-C:H sputter yield fall between 2.3 to 2.8 carbon atoms per incident ion at an acceleration voltage of $50 \mathrm{kV}$. ${ }^{16}$ Theoretically determined values for carbon sputter yield, however, range from 1.2 to 1.7 at an acceleration voltage of $30 \mathrm{kV}$, with the former obtained by transport of ions in matter (TRIM) simulations ${ }^{18}$ and the latter through use of the linear cascade collision (LCC) model developed by Sigmund. ${ }^{17}$ We refrain from using the computationally determined sputter yields due to the limitations associated with these models. For example, the TRIM simulations do not take into account any orientation dependent phenomena such as the channeling of $\mathrm{Ga}^{+}$ions through the graphene lattice. The LCC model, whose underlying assumptions require ample material volume and an amorphous structure, is also unlikely applicable in estimating the sputtering yield of graphene due to its $2 \mathrm{D}$ nature and highly ordered lattice. ${ }^{17}$ Furthermore, we find that even if the theoretically determined sputter yields were taken into account, they would have marginal influence on our subsequent calculations for the estimated graphene sputter time. 


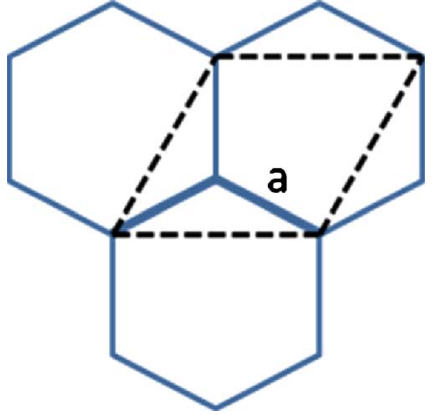

FIG. 5. (Color online) Unit cell of the graphene lattice outlined by the dashed lines: two atoms and three bonds are included. $\mathrm{a}=1.42 \AA$.

We determine the $\mathrm{Ga}^{+}$ion flux incident on the a-C:H film (and, therefore, on graphene) by calculating the total number of sputtered carbon atoms from a selected area, and then dividing it by both the sputter yield and the time required to fully etch away the film in that area. First, by assuming the mass density of the a-C:H film to be $2 \mathrm{~g} / \mathrm{cm}^{3}$, as widely reported for amorphous carbon films, ${ }^{19}$ we determine the number of sputtered $\mathrm{C}$ atoms, $\mathrm{N}_{\mathrm{s}}$, from the $125 \mathrm{~nm}$ thick a-C:H film to be $1.254 \times 10^{18} \mathrm{C}$ atoms $/ \mathrm{cm}^{2}$ by utilizing Eq. (1)

$$
\mathrm{N}_{\mathrm{S}}=\frac{\rho_{\mathrm{C}} \times \mathrm{N}_{\mathrm{A}} \times \mathrm{d}}{\mathrm{m}_{\mathrm{c}}},
$$

where $\rho_{\mathrm{C}}$ is the mass density of the a-C:H film, $\mathrm{N}_{\mathrm{A}}$ is Avogadro's Number, $m_{c}$ is atomic mass of carbon, and $d$ is the film thickness. Using the experimentally-determined etch time, $t_{\text {etch }}$, required to entirely remove the film in the $4.33 \mu \mathrm{m}^{2}$ area, we calculate the sputter rate $\left(\mathrm{R}_{\mathrm{S}}\right)$ to be $2.786 \times 10^{16}$ atoms $/ \mathrm{cm}^{2}$ s by using Eq. (2)

$$
\mathrm{R}_{\mathrm{S}}=\mathrm{N}_{\mathrm{S}} / \mathrm{t}_{\text {etch }} .
$$

We then determine the $\mathrm{Ga}^{+}$flux $\left(\mathrm{J}_{\mathrm{Ga}^{+}}\right)$to be $1.059 \times 10^{16} \mathrm{Ga}^{+}$ (ions $/ \mathrm{cm}^{2}$ sthrough Eq. (3)

$$
\mathrm{J}_{\mathrm{Ga}^{+}}=\mathrm{R}_{\mathrm{S}} / \mathrm{Y}_{\mathrm{a}-\mathrm{C}: \mathrm{H}} .
$$

We then use the carbon-carbon bond length of $1.42 \AA$ and the geometry of the graphene lattice, Fig. 5, to calculate the aerial density of graphene $\left(\mathrm{A}_{\rho}\right)$ to be 3.818 $\times 10^{15}$ atoms $/ \mathrm{cm}^{2}$. If the sputtering mechanism in graphene is similar to that in other carbon-based structures, the theoretical time $\left(t_{\text {theo }}\right)$ to remove a single graphene sheet of a certain area in its entirety can be calculated by

$$
\mathrm{t}_{\text {theo }}=\frac{\mathrm{A}_{\rho}}{\frac{\mathrm{A}_{\mathrm{a}-\mathrm{C}: \mathrm{H}}}{\mathrm{A}_{\mathrm{S}}} \times \mathrm{J}_{\mathrm{Ga}^{+}} \times \mathrm{Y}_{\mathrm{a}-\mathrm{C}: \mathrm{H}}},
$$

here $\mathrm{A}_{\mathrm{a}-\mathrm{C}: \mathrm{H}} / \mathrm{A}_{\mathrm{S}}$ is the ratio between the exposed area of the $\mathrm{a}-\mathrm{C}: \mathrm{H}$ film $\left(\mathrm{A}_{\mathrm{a}-\mathrm{C}: \mathrm{H}}=4.33 \mu \mathrm{m}^{2}\right)$ and the total area $\left(\mathrm{A}_{\mathrm{S}}\right)$ of the image depicting a graphene monolayer sample. This scaling takes into account the frequency with which the ion beam rasters over the exposed area. For the graphene monolayer presented in Fig. 3(a) (sample 1), $\mathrm{A}_{\mathrm{S} 1}=290.1 \mu \mathrm{m}^{2}$, and $t_{\text {theo }}=8.96 \mathrm{~s}$, resulting in the ratio between $t_{\text {exp }}$ and $t_{\text {theo }}$ to be 6.362. For the second graphene monolayer studied here
TABLE I. Sputtering times for single-layer graphene. (The average of $t_{\text {exp }} / t_{\text {theo }}$ is 7.03.)

\begin{tabular}{ccccc}
\hline \hline Sample & $\mathrm{A}_{\mathrm{a}-\mathrm{C}: \mathrm{H}} / \mathrm{A}_{\mathrm{S}}$ & $\begin{array}{c}\mathrm{t}_{\text {theo }} \\
(\mathrm{s})\end{array}$ & $\begin{array}{c}\mathrm{t}_{\exp } \\
(\mathrm{s})\end{array}$ & $\mathrm{t}_{\text {exp }} / \mathrm{t}_{\text {theo }}$ \\
\hline 1 & 0.0153 & 8.96 & 57.0 & 6.362 \\
2 & 0.1290 & 1.06 & 8.15 & 7.689 \\
\hline \hline
\end{tabular}

(sample 2), $\mathrm{A}_{\mathrm{S} 2}=34.3 \mu \mathrm{m}^{2}, \mathrm{t}_{\text {theo }}=1.06$, and $\mathrm{t}_{\mathrm{exp}}=8.15 \mathrm{~s}$. Using the $t_{\exp } / t_{\text {theo }}$ ratios for these two samples, we calculate that average $t_{\exp }$ is $\sim 7.03$ times longer than $t_{\text {theo }}$ for a single sheet of graphene (Table I). This $t_{\text {exp }}$ surpasses any expectations for a carbon-based material under ion bombardment.

We believe that this resilience stems from the 2D nature of graphene. Since graphene is only one atom thick, it eliminates the volume in which sputtering cascade collisions occur in bulk materials and minimizes the interactions with recoiling atoms from the underlying substrate and graphene lattice. ${ }^{17,20,21}$ The absence of cascade collisions near the surface would greatly reduce the number of ejected carbon atoms from the graphene lattice. It is also possible that $\mathrm{C}$ atoms in graphene act as an intermediary in transferring the kinetic energy to the underlying substrate without being readily displaced from the lattice, acting like a system of billiard balls where energy is completely transferred to the substrate underneath. Graphene's relatively open and ordered atomic structure may also facilitate $\mathrm{Ga}^{+}$ion channeling through its lattice. ${ }^{17,22}$ This would limit the number of primary ion collisions, thus reducing the number of carbon atoms sputtered away. Ultimately, $\mathrm{Ga}^{+}$ions that are not backscattered by the graphene lattice come to a halt in the underlying $\mathrm{SiO}_{2}$ after losing their kinetic energy through elastic and inelastic collisions. Since $\mathrm{SiO}_{2}$ is an insulator, $\mathrm{Ga}^{+}$ions may be implanted into the substrate surface, causing a net positive charge to build up in the region exposed to ion bombardment, forcing the subsequent incoming incident ions to be electrostatically repelled. ${ }^{18}$ These considerations should be taken into account when developing a more thorough understanding of graphene's ability to withstand a prolonged ion beam exposure.

Further investigations, involving the substitution and elimination of the substrate, will help shed light onto the specific mechanisms operating during sputtering of graphene by removing any effects that the substrate has on the cascade collision process. Elimination of the substrate will ensure that mechanisms inducing the sputtering of graphene will be solely from elastic and inelastic collisions with incident ions.

\section{CONCLUSION}

In summary, we convincingly demonstrate the ability of a single-atom-thick graphene layer to withstand approximately seven times higher ion bombardment than expected. Our findings reaffirm the wide range of science that is yet to be done on this unique single-atom thick material. 


\section{ACKNOWLEDGMENTS}

We gratefully acknowledge financial support of the NRI INDEX Center. We thank M. J. Burek for useful discussions and assistance with the DualBeam system. Access to the Dual-Beam System was provided by the Kavli Nanoscience Institute (KNI) at Caltech. We also appreciate assistance from E. Miura and G. R. Rossman with Raman Spectroscopy, and we thank C. Daraio for access to the optical microscope.

${ }^{1}$ K. S. Novoselov, A. K. Geim, S. V. Morozov, D. Jiang, Y. Zhang, S. V. Dubonos, I. V. Grigorieva, and A. A. Firsov, Science 306, 666 (2004).

${ }^{2}$ A. K. Geim and K. S. Novoselov, Nature Mater. 6, 183 (2007).

${ }^{3}$ K. S. Novoselov, A. K. Geim, S. V. Morozov, D. Jiang, M. I. Katsnelson, I. V. Grigorieva, S. V. Dubonos, and A. A. Firsov, Nature (London) 438, 197 (2005).

${ }^{4}$ Y. Zhang, Y.-W. Tan, H. L. Stormer, and P. Kim, Nature (London) 438, 201 (2005).

${ }^{5}$ K. I. Bolotin, K. J. Sikes, Z. Jiang, M. Klima, G. Fudenberg, J. Hone, P. Kim, and J/ L. Stormer, Solid State Commun. 146, 351 (2008).

${ }^{6}$ C. Lee, X. Wei, J. W. Kysar, and J. Hone, Science 321, 385 (2008).

${ }^{7}$ K. S. Kim, Y. Zhao, H. Jang, S. Y. Lee, J. M. Kim, K. S. Kim, J.-H. Ahn, P. Kim, J.-Y. Choi, and B. H. Hong, Nature (London) 457, 706 (2009).

${ }^{8}$ D. Teweldebrhan and A. A. Balandin, Appl. Phys. Lett. 94, 013101 (2009).
${ }^{9}$ K. S. Novoselov, D. Jiang, F. Schedin, T. J. Booth, V. V. Khotkevich, S. V. Morozov, and A. K. Geim, Proc. Natl. Acad. Sci. U.S.A. 102, 10451 (2005).

${ }^{10}$ R. R. Nair, P. Blake, A. N. Grigorenko, K. S. Novoselov, T. J. Booth, T. Stauber, N. M. R. Peres, and A. K. Geim, Science 320, 1308 (2008).

${ }^{11}$ P. Blake, E. W. Hill, A. H. Neto Castro, K. S. Novoselov, D. Jiang, R. Yang, T. J. Booth, and A. K. Geim, Appl. Phys. Lett. 91, 063124 (2007).

${ }^{12}$ A. C. Ferrari, J. C. Meyer, V. Scardaci, C. Casiraghi, M. Lazzeri, F. Mauri, S. Piscanec, D. Jiang, K. S. Novoselov, S. Roth, and A. K. Geim, Phys. Rev. Lett. 97, 187401 (2006).

${ }^{13}$ A. Gupta, G. Chen, P. Joshi, S. Tadigadapa, and P. C. Eklund, Nano Lett. 6, 2667 (2006).

${ }^{14}$ M. A. Tamor and W. C. Vassel, J. Appl. Phys. 76, 3823 (1994).

${ }^{15}$ A. C. Ferrari and J. Robertson, Phys. Rev. B 61, 14095 (2000).

${ }^{16}$ A. Stanishevsky, Thin Solid Films 398-399, 560 (2001).

${ }^{17} \mathrm{~J}$. Orloff, M. Utlaut, and L. Swanson, High Resolution Focused Ion Beams: FIB and Its Applications (Kluwer/Plenum, New York, 2003).

${ }^{18}$ Introduction to Focused Ion Beams: Instrumentation, Theory, Techniques, and Practice, edited by L. A. Giannuzzi and F. A. Stevie (Springer, New York, 2005), pp. 13-101.

${ }^{19}$ CRC Handbook of Chemistry and Physics (CRC, Cleveland, 1979).

${ }^{20}$ Ion-Solid Interactions: Fundamentals and Applications, in edited by M. Nastasi, J. W. Mayer, and J. K. Hirvonen (Cambridge University Press, Cambridge, 1996).

${ }^{21}$ Sputtering by Particle Bombardment: Experiments and Computer Calculations from Threshold to MeV Energies, edited by R. Behrisch and W. Eckstein (Springer, Berlin, 2007).

${ }^{22}$ C. A. Volkert and A. M. Minor, Mater. Res. Bull. 32, 389 (2007). 